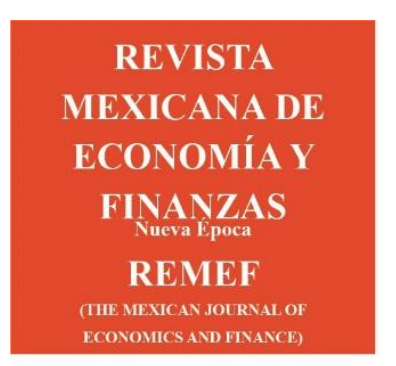

Revista Mexicana de Economía y Finanzas, Nueva Época

Volumen 16 Número 3, Julio - Septiembre 2021, pp. 1-18, e650

THE ECONOMICS AND FINANCE EFFECTS OF THE COVID-19 PANDEMIC Editor: Dr. Ignacio Perrotini

DOI: https://doi.org/10.21919/remef.v16i3.650

(Recibido: $1 \%$ febrero/2021, aceptado: 4/junio/2021, publicado: 10/junio/2021)

\title{
Afectaciones financieras en los principales países de América Latina con mayores registros de COVID-19
}

\author{
Héctor Alonso Olivares Aguayo ${ }^{1}$ - Universidad La Salle, México
}

El objetivo de la investigación es determinar las afectaciones que ha causado la pandemia COVID-19 en los principales mercados de América Latina (Brasil, Argentina, Colombia y México). Se realiza un análisis del Valor en Riesgo a diferentes niveles de confianza para la conformación de portafolios de inversión óptimos del tipo Markowitz y Sharpe en los componentes del BOVESPA, S\&P Merval, COLCAP y S\&P BMV/ IPC. Los resultados muestran que para ambos tipos de portafolios las mayores pérdidas esperadas las tiene el mercado argentino y las menores el mexicano. Por lo que de las economías analizadas en esta investigación es más recomendable invertir en el mercado accionario de México. Como limitación se asume el supuesto de rendimientos gaussianos. El trabajo es original porque los datos históricos diarios consideran el periodo del COVID-19. Se concluye que, a pesar, que Brasil es el país con mayor número de contagios por COVID-19 de América Latina, con base en la métrica de Valor en Riesgo, Argentina es el más afectado financieramente y México el menor.

Clasificación JEL: G11, G12, I18.

Palabras clave: COVID-19, Teoría del Portafolio, rendimientos.

\section{Financial impacts in the main Latin American countries with the highest records of COVID-19}

The objective of the investigation is to determine the effects that the COVID-19 pandemic has caused in the main markets of Latin America (Brazil, Argentina, Colombia and Mexico). An analysis of the Value at Risk is carried out at different levels of confidence for the conformation of optimal investment portfolios of the Markowitz and Sharpe type in the components of BOVESPA, S\&P Merval, COLCAP and S\&P BMV / IPC. The results show that for both types of portfolios the highest expected losses are in the Argentine market and the lowest in the Mexican market. Therefore, of the economies analyzed in this research, it is more advisable to invest in the Mexican stock market. As a limitation, the assumption of Gaussian returns is assumed. The work is original because the daily historical data considers the period of COVID-19. It is concluded that although Brazil is the country with the highest number of COVID-19 infections in Latin America, based on the Value at Risk metric, Argen tina is the most affected financially and Mexico the least.

JEL Classification: G11, G12, I18.

Keywords: COVID-19, Portfolio Theory, Returns.

\footnotetext{
${ }^{1}$ Autor de correspondencia. ORCID: https://orcid.org/0000-0003-2609-8627

$*$ Sin fuente de financiamiento para el desarrollo de la investigación
} 


\section{Introducción}

El COVID-19 surgió a finales del año 2019 en la ciudad de Wuhan, China como se muestra en la Imagen 1.

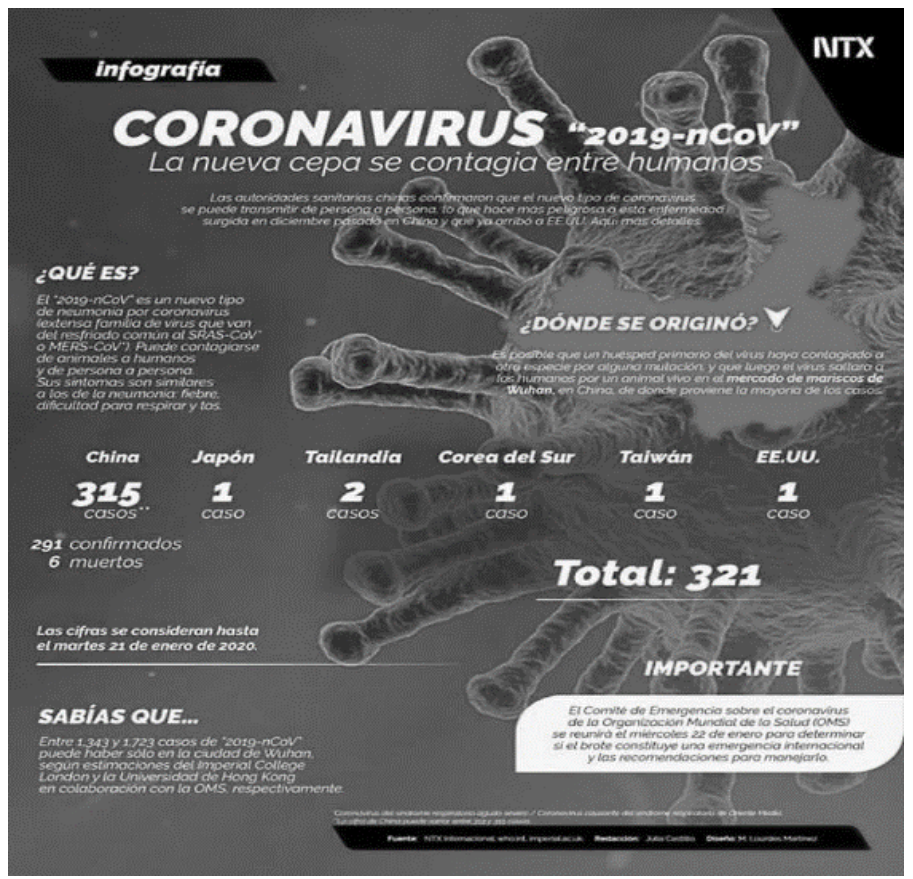

Imagen 1. Surgimiento del COVID-19

Fuente: CONAPRENC, 2020.

Las cifras mostradas en la Imagen 1, se han elevado en gran medida al día de hoy 30 de abril de 2021, prácticamente un año y cuatro meses después se tienen más de 151 millones de contagios a nivel mundial, más de 3 millones de defunciones y la mayor cantidad de nuevos casos diarios superando los 900 mil, justo una semana antes, el día 23 de abril del presente año. Particularmente el país con mayor afectación ha sido los Estados Unidos con más de 33 millones de casos totales y más de 589 mil defunciones. Prácticamente el 21.85\% y el $19.63 \%$ respectivamente de las cifras mundiales. En América Latina los países más afectados han sido: Brasil con más de 14 millones de casos totales y más de 400 mil defunciones, le sigue Argentina con más de 2.9 millones de casos totales y más de 63 mil defunciones, inmediatamente después se encuentra Colombia con más de 2.8 millones de casos totales y más de 73 mil defunciones, y le sigue México con más de 2.3 millones de casos totales y más de 216 mil defunciones; en conjunto estos países engloban más de 22 millones de casos totales y más de 752 mil defunciones. Prácticamente el $14.57 \%$ y el 25.06\% respectivamente de las cifras mundiales. Lo anterior se muestra en la Imagen 2. 


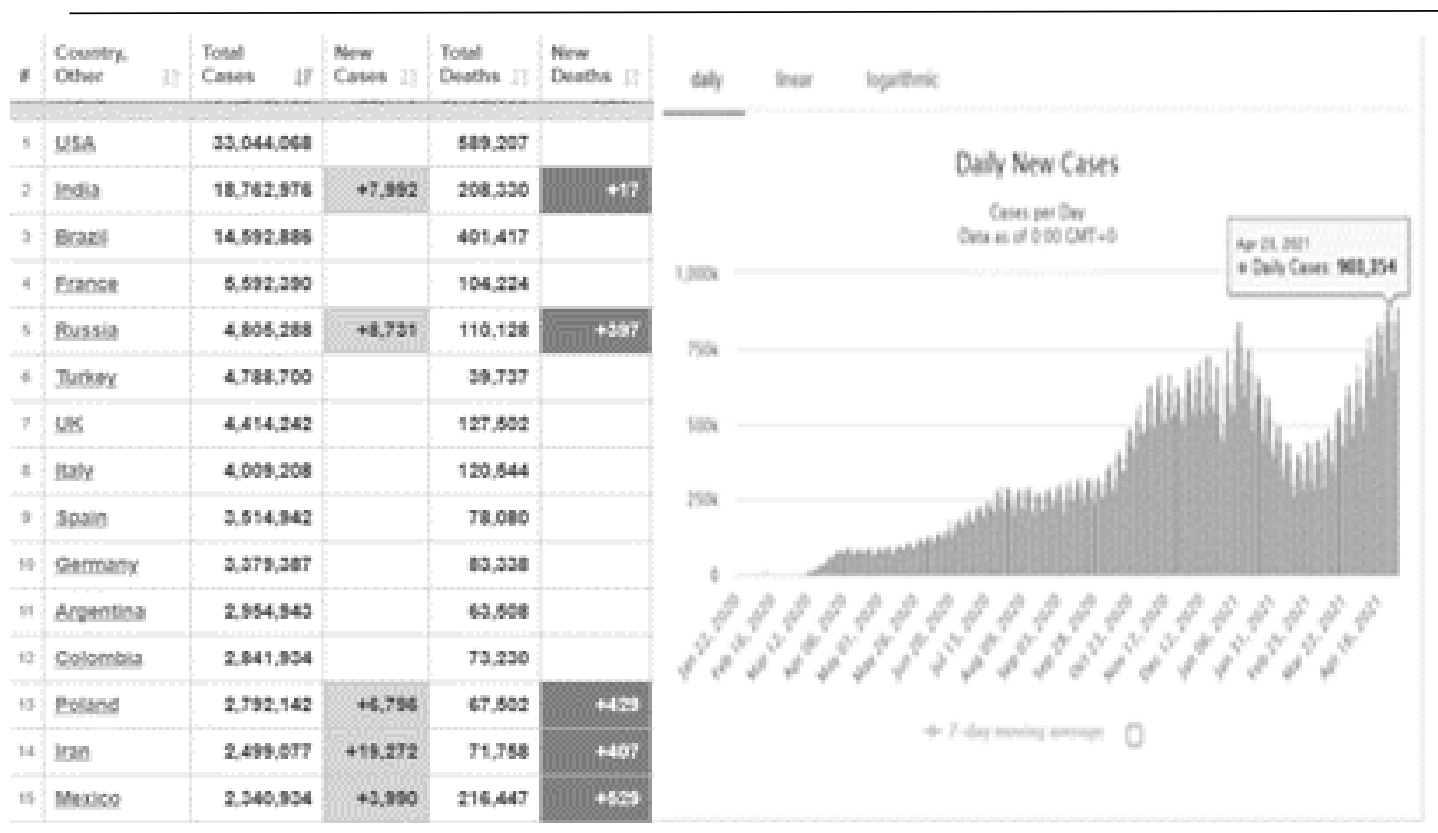

Imagen 2. Resumen de la situación mundial del COVID-19 al 30 de abril de 2021

Fuente: worldometers, 2021.

El COVID-19 ha impactado a muchas economías y América Latina no es la excepción. Algunas investigaciones recientes sobre este tipo de economías se pueden apreciar en Martínez y Parraguez (2021), quienes muestran los daños que se han tenido en las economías de Chile y Colombia y como éstas se han potencializado a raíz de la pandemia COVID-19, por su parte Mendoza et al. (2020). Examinan el impacto de la pandemia COVID-19 a través del índice de letalidad sobre los índices bursátiles, tasas de interés y tipos de cambio de: México, Chile, Perú, Colombia, Brasil y Argentina. Esta investigación tiene como objetivo determinar las afectaciones que ha causado la pandemia COVID-19 en los principales mercados de América Latina (Brasil, Argentina, Colombia y México), bajo la hipótesis de que la métrica del Valor en Riesgo (VaR) con diferentes niveles de confianza en los portafolios de inversión óptimos de Markowitz y Sharpe asociados a los componentes de los índices respectivos, dan síntomas de las pérdidas financieras potenciales que pudieran llegar a tener los inversionistas, siendo el país con mayor pérdida esperada el más afectado y el de menor el menos. La investigación está organizada de la siguiente manera: en la sección 2 se presenta una breve revisión de literatura, seguida de la sección 3 donde se presentan las metodologías de los portafolios de inversión óptimos y del VaR Delta-Normal, en la sección 4 se presentan los resultados obtenidos, y por último en la sección 5 se concluye.

\section{Revisión de Literatura}

La teoría de los mercados eficientes, expuesta por Fama (1965), supone distintos tipos de eficiencia en los mercados financieros por el cambio aleatorio que tienen los precios dependiendo del grado de información con la cual los agentes económicos racionales disponen, estos tipos de eficiencia pueden ser: débil, semi-fuerte o fuerte. Particularmente para que se dé esta última, todos los participantes deben de tener acceso a la misma información, sin embargo, este tipo de eficiencia no existe, pues dichos agentes poseen información de privilegio. 
En los mercados financieros no existe competencia perfecta. Es decir, no hay previsión perfecta, ya que se genera una mayor incertidumbre e incrementa el riesgo para este agente y para las empresas, porque la información realmente es asimétrica, y las decisiones que se toman de esta manera tal vez no sean las más acertadas, por lo anterior es importante realizar un análisis de administración de riesgos en estos tipos de mercados. Estas teorías ayudan a crear técnicas, políticas, y nuevos instrumentos aplicados en mejora del desempeño de los mercados financieros, es decir la innovación de productos financieros a través de la ingeniería financiera ayudan a los agentes racionales a tomar decisiones óptimas de maximización de beneficio y minimización de riesgos en sus inversiones. Lo cual es el objetivo primordial de la teoría moderna de selección de portafolios de inversión de Markowitz (1952), trabajo seminal de la economía financiera y uno de los aportes teóricos de las ciencias económicas más relevantes.

Un portafolio eficiente, según Harry Markowitz, es aquel que tiene un mínimo riesgo, para un rendimiento dado (modelo media-varianza), en el que se demuestra que los agentes económicos son racionales al considerar una cartera compuesta por la diversificación de activos.

Byder, et al. (2019), muestran estudios de la reacción de los agentes económicos racionales en crisis financiera. Preciado y Camacho (2009), aplican la metodología de selección de portafolios óptimos de inversión del tipo Markowitz a partir de diferentes alternativas pensionarias. León, et al. (2015), proponen el modelo de Estrada (media-semivarianza), para un portafolio con activos financieros de agricultura. Algunas investigaciones con portafolios aplicadas en América Latina se muestran en Mora, et al. (2010), quienes utilizan cuatro métodos distintos en las siete Bolsas de Valores más relevantes de América Latina en el periodo 2001-2006. Mientras que Luna y Agudelo (2019), usan el modelo Black-Litterman con datos trimestrales en Portafolios accionarios de los países del MILA: Colombia, Chile, México y Perú. Agudelo, et al. (2021), muestran un comparativo de portafiolios de renta fija respecto a los de renta variable, mostrando empíricamente mayor rentabilidad para los portafolios de renta fija previo a la crisis COVID-19 para el caso mexicano, Arévalo (2015) realiza un análisis de sentimientos, para concluir que la asignación óptima de varianza media no necesariamente corresponde a los encontrados bajo supuestos del comportamiento. Estrada y Toro (2014), mediante un sistema experto, predicen la variabilidad del portafolio de Markowitz.

Álvarez, et al. (2017), evalúan el desempeño de tres modelos de estructura de plazos dinámicos de tasas de interés para estimar el Valor en Riesgo (VaR) de los portafolios de renta fija. Gutiérrez, et al. (2017), resuelven el problema clásico de optimización del portafolio, calculando la volatilidad a través de los modelos de heterocedasticidad condicional autorregresiva generalizada y García, et al. (2019), analizan las medidas de riesgo tradicionales usadas en modelos de optimización del portafolio. 


\section{Metodología}

\subsection{Planteamiento del modelo media-varianza}

El modelo de media-varianza supone que los agentes económicos racionales toman sus decisiones de inversión solamente considerando dos variables en la conformación de sus portafolios: rendimiento esperado y varianza.

Este tipo de agentes prefieren un mayor rendimiento esperado a igual nivel de riesgo en un par de inversiones, y a su vez a igual rendimiento esperado prefieren la del menor riesgo.

"Debido a que Markowitz utiliza el binomio rentabilidad-riesgo, y riesgo medido por la varianza, se suele conocer a su modelo como el enfoque media-varianza” (Brun y Moreno, 2008, pág. 33).

El modelo media-varianza permite encontrar las combinaciones de activos que tienen varianza y por ende desviación estándar mínima con distintas tasas de rendimiento esperadas, lo cual genera la curva de mínima varianza; análogamente se pueden encontrar aquellas combinaciones que producen valores esperados de rendimiento máximo para diferentes niveles de varianza dados. El conjunto de combinaciones de activos de inversión que reúnen estas dos propiedades en forma simultánea se conoce como la "frontera eficiente de oportunidades de inversión". Por lo que, el enfoque de Markowitz hace posible que sean descartadas un gran número de canastas de consumo de riesgo y rendimiento que se encuentran por debajo de la frontera eficiente.

La suma ponderada de los rendimientos esperados $E\left(R_{i}\right)$ de cada activo $w_{i}$ con $i=$ $1,2, \ldots \ldots, n$, representa el rendimiento esperado del portafolio de inversión $E\left(R_{p}\right)$, expresado matemáticamente de la manera siguiente:

$$
E\left(R_{p}\right)=\sum_{i=1}^{n} w_{i} E\left(R_{i}\right), \quad w_{i} \geq 0
$$

La condición $w_{i} \geq 0$ limita las ventas en corto. Los precios de los activos elegidos deben ser diarios, pero como los modelos de la teoría financiera no trabajan con precios, sino con rendimientos continuos, entonces se realiza el cálculo de los rendimientos como el logaritmo natural de la razón entre el precio de cierre del día actual y el anterior.

$$
\ln \left(\frac{P_{t}}{P_{t-1}}\right)
$$

Siendo $P_{t}$ es el precio del activo en el periodo $t$ y $P_{t-1}$ el precio del activo en el periodo $t$ - 1 . El riesgo del portafolio de inversión se representa con $\operatorname{Var}\left(R_{p}\right)$ que es la varianza de los rendimientos del portafolio. La fórmula en términos del valor esperado es:

$$
=\sum_{i=1}^{n} \sum_{j=1}^{n} w_{i} w_{j} \operatorname{Cov}\left(R_{i}, R_{j}\right)
$$




$$
=\sum_{i=1}^{n} \sum_{j=1}^{n} w_{i} w_{j} \sigma_{i j}
$$

Donde $\sigma_{i j}$ es la covarianza entre los rendimientos de los activos $i$ y $j$, definida como $\sigma_{i j}=$ $E\left[R_{i}-E\left(R_{i}\right)\right]\left[R_{i}-E\left(R_{j}\right)\right]$, expresada en términos del coeficiente de correlación lineal $\rho_{i j}$, equivale a:

$$
\operatorname{Var}\left(R_{p}\right)=\sum_{i=1}^{n} \sum_{j=1}^{n} w_{i} w_{j} \rho_{i j} \sigma_{i} \sigma_{j}
$$

La correlación es una medida estadística que es útil en finanzas para determinar el tipo de relación directa o inversa entre los rendimientos de dos activos, dependiendo el signo que tenga, como se muestra en la siguiente ecuación:

$$
-1 \leq \rho_{i j} \leq 1
$$

Así el agente económico racional puede elegir entre diferentes tipos de combinaciones o canastas de rendimiento y riesgo, dadas sus expectativas que tenga sobre $E\left(R_{p}\right)$ y $\operatorname{Var}\left(R_{p}\right)$.

\subsection{Problema de optimización}

Como se ha mencionado, el agente económico racional busca maximizar su bienestar, sujeto a las limitantes de sus recursos (dinero y tiempo). Entre más grande sea dicho bienestar esto le provocará una mayor felicidad o satisfacción ante las distintas posibilidades de consumo derivadas de su restricción presupuestal y dadas sus preferencias de niveles de riesgo y rendimiento en la selección de sus portafolios de inversión, por lo anterior, el consumidor inversionista racional optará por la combinación de bienes que le proporcione la máxima utilidad, es decir, satisface el principio de insaciabilidad, pues se tiene el deseo de siempre buscar una mayor satisfacción, es decir no se tiene un punto de saciedad.

El planteamiento al modelo de optimización de Markowitz (gráficamente se encuentra en el vértice de la parábola, donde en el eje vertical corresponde al rendimiento esperado y el horizontal al riesgo) minimiza la varianza del rendimiento del portafolio y por ende el riesgo, dado un cierto nivel de rendimiento esperado como se aprecia a continuación:

$$
\begin{gathered}
\min \operatorname{Var}\left(R_{p}\right)=\sum_{i=1}^{n} \sum_{j=1}^{n} w_{i} w_{j} \sigma_{i j} \\
=\left(w_{1}, w_{2}, \cdots \cdots, w_{n}\right)\left(\begin{array}{ccccc}
\sigma_{11} & \sigma_{12} & \cdots & \cdots & \sigma_{1 n} \\
\sigma_{21} & \ddots & & & \\
\vdots & & & & \\
\sigma_{n 1} & \cdots & \cdots & & \sigma_{n n}
\end{array}\right)\left(\begin{array}{c}
w_{1} \\
w_{2} \\
\vdots \\
w_{n}
\end{array}\right)
\end{gathered}
$$

sujeto a: 


$$
\begin{gathered}
E\left(R_{p}\right)=\sum_{i=1}^{n} w_{i} E\left(R_{i}\right) \\
\sum_{i=1}^{n} w_{i}=1
\end{gathered}
$$

La varianza del rendimiento del portafolio se minimiza con la primera restricción, que muestra un promedio ponderado del rendimiento esperado de cada activo, y la segunda restricción indica que la suma de las ponderaciones a considerar en el portafolio es el $100 \%$.

$\mathrm{Al}$ resolver el problema, se encuentran las ponderaciones óptimas de inversión $w_{i}{ }^{*}$ de cada activo del portafolio que satisfacen ambas restricciones del modelo.

Investigaciones posteriores a las de Markowitz incorporan un activo libre de riesgo, por ejemplo, Tobin (1958), Treynor (1962), Sharpe (1963) y (1970), Lintner (1965) y Mossin (1966) muestran que si los inversionistas pueden prestar y pedir prestado a la tasa de interés "libre de riesgo" se obtiene una región eficiente lineal superior a la frontera eficiente de Markowitz.

En este caso, el rendimiento esperado del portafolio tiene como benchmark el rendimiento "libre de riesgo", siendo una función lineal de la relación entre el riesgo del mercado y del portafolio, medido a través de su desviación estándar. Es decir, con este modelo los rendimientos de otros activos en condiciones sin crisis deberán ofrecer una prima por el riesgo al que está expuesto el agente económico racional sobre el rendimiento ofrecido por el activo "libre de riesgo" de incumplimiento; además de que dicha prima depende del nivel de riesgo que el activo mantiene con el riesgo del mercado.

$$
E\left(R_{p}\right)=T L R+\left(\frac{E\left(r_{m}\right)-T L R}{\sigma_{m}}\right) * \sqrt{\operatorname{Var}\left(R_{p}\right)}
$$

$T L R$ : Rendimiento del activo libre de riesgo.

$E\left(r_{m}\right)$ : Rendimiento esperado del mercado.

$\sigma_{m}$ : Desviación estándar del mercado.

$\sqrt{\operatorname{Var}\left(R_{p}\right)}$ : Desviación estándar del portafolio.

$E\left(R_{p}\right)$ : Rendimiento esperado del portafolio.

La pendiente de la línea del mercado de capitales $\left(E\left(r_{m}\right)-T L R\right) / \sigma_{m}$ representa el precio del riesgo, mejor dicho, el rendimiento adicional por cada unidad de riesgo.

Otra manera de ver (7) es:

$$
E\left(R_{p}\right)=T L R+\beta_{i}\left(E\left(r_{m}\right)-T L R\right)
$$

$\beta_{i}$ : Medida de riesgo beta del activo i-ésimo.

La relación entre el coeficiente beta de un activo y su correlación con el mercado está dada por: 


$$
\beta_{i m}=\frac{\rho_{i m} \sigma_{i} \sigma_{m}}{\sigma_{m}^{2}}
$$

$\beta_{\text {im }}$ : Coeficiente beta entre el activo i y el mercado.

$\rho_{i m}$ : Coeficiente de correlación entre el activo i y el mercado.

$\sigma_{i}$ : Desviación estándar del activo i-ésimo.

$\sigma_{m}$ : Desviación estándar del mercado.

$\sigma_{m}^{2}$ : Varianza del mercado.

El coeficiente beta al ser un indicador global del riesgo del portafolio, simplifica el análisis de los activos, identificando fácilmente los activos poco riesgosos cuando sus betas tienen valores menores a uno, mientras que cuando sean mayores a la unidad, los activos serán riesgosos.

Cabe señalar que al optimizar el portafolio que compensa de mejor forma la relación riesgorendimiento conocido como el portafolio de Sharpe, el cual hace tangencia con la frontera eficiente de Markowitz, el único cambio que se hace en el planteamiento del problema de optimización en la función objetivo es maximizar el índice de Sharpe $(I S)$ el cual se expresa de la forma siguiente:

$$
I S=\frac{E\left(R_{p}-T L R\right)}{\sqrt{\operatorname{Var}\left(R_{p}\right)}}
$$

\subsection{Frontera eficiente del portafolio de inversión}

La frontera eficiente del portafolio de inversión en términos de un problema de investigación de operaciones "primal" es la combinación de activos que, dado un nivel de rendimiento, proporcionan el mínimo riesgo y de forma "dual” para un nivel dado de riesgo, proporciona el máxima rendimiento.

El conjunto de portafolios óptimos será el que se localice limitado por el rendimiento esperado mínimo y máximo de cada activo que componga el portafolio, es decir, si $M=$ $\max \left[E\left(R_{i}\right): i=1, \ldots, n\right]$ y $m=\operatorname{mín}\left\{E\left(R_{i}\right): i=1, \ldots, n\right\}$ entonces $E\left(R_{p}\right) \in[m, M]$.

Para hallar el portafolio óptimo sobre la frontera eficiente de este modelo se debe encontrar el punto sobre el que la función de utilidad del inversionista hace tangencia con dicha frontera.

En cuanto al cálculo del VaR paramétrico se hace uso del tipo Delta-Normal como se muestra en Olivares, et al. (2017).

$$
\operatorname{VaR}_{z}^{(1-\alpha) \%}=Z_{(1-\alpha) \%}\left\{\left(w_{1}, w_{2}, \ldots, w_{n}\right)\left[\begin{array}{cccc}
\sigma^{2}{ }_{1} & \sigma_{12} & \ldots & \sigma_{1 n} \\
\sigma_{21} & \sigma^{2}{ }_{2} & \ldots & \sigma_{2 n} \\
\cdot & . & \ldots & \cdot \\
\cdot & & & \\
\sigma_{n 1} & \sigma_{n 2} & \ldots & \sigma_{n}^{2}
\end{array}\right]\left[\begin{array}{c}
w_{1} \\
w_{2} \\
\cdot \\
\cdot \\
w_{n}
\end{array}\right] * t\right\}^{\frac{1}{2}}
$$

Donde, $\sigma_{i j}$ : Varianza entre los rendimientos de los activos i y j si $i=j$ o bien Covarianza si $i \neq j$. 
$Z_{(1-\alpha) \%}$ : Cuantil de una distribución gaussiana con nivel de confianza de $(1-\alpha) \%$.

$\alpha$ : Nivel de significancia, $0 \leq \alpha \leq 1$.

$t$ : Horizonte temporal.

\section{Resultados}

En esta investigación se realizaron los cálculos de diversos portafolios de inversión a través de la herramienta solver de Excel, analizando cuatro portafolios del tipo Markowitz y cuatro de Sharpe, a través de la diversificación de activos de los índices de los principales mercados financieros de América Latina (BOVESPA, S\&P MERVAL, COLCAP y S\&P BMV/IPC) que han sido mayormente afectados por la pandemia COVID-19, estos mercados corresponden a los países de: Brasil, Argentina, Colombia y México, se consideraron datos históricos diarios correspondientes al periodo 12/02/2019 al 12/02/2021, lo cual representa una estadística robusta, posteriormente se calcularon estadísticos descriptivos básicos de los rendimientos de cada componente del índice respectivo y del mismo índice, se realizó el proceso correspondiente para la construcción tanto de la frontera eficiente como de los portafolios óptimos de mínima varianza de Markowitz y Sharpe, éstos últimos fueron comparados con el riesgo y rendimiento que ofrece el mercado de cada país analizado de América Latina. Una vez que se encontraron los parámetros de riesgo y rendimiento en los portafolios se procede al cálculo del VaR paramétrico Delta-Normal a los niveles de confianza recomendados por el Comité de Basilea: 95\%, 99\% y 99.9\%, ya que cada vez éstos niveles son más restrictivos desde Basilea II a IV como se puede apreciar en Rossignolo (2019).

El proceso para la conformación de los portafolios para cada país fue el siguiente:

I) Se descargaron del sitio web de investing, los precios de cierre diarios de cada índice y de sus componentes, para la construcción de la base de datos, ésta fue armada únicamente con los componentes con misma cotización que su índice en el periodo seleccionado.

II) Se calcularon los rendimientos diarios del tipo continuo para cada activo y se analizaron sus estadísticos descriptivos básicos en términos anuales.

III) El criterio de selección para la conformación de portafolios fue el $I S$, el cual, se consideró de forma positiva, es decir, aquellos activos con $I S<0$, fueron descartados inmediatamente como candidatos en la conformación del portafolio, pues su rendimiento esperado se encontraba por debajo de su tasa de referencia establecida por el Banco Central respectivo de cada país. Cabe decir que se trabajó con las tasas de referencia: SELIC para Brasil, la tasa de política monetaria para Argentina, TES para Colombia y TIIE para México.

IV) Posteriormente se procedió a la obtención de la matriz de varianzas y covarianzas.

V) Se implementó el optimizador solver de Excel en los modelos de Markowitz y Sharpe, determinando así las ponderaciones para cada activo en la conformación del portafolio.

VI) Se realizó el cálculo del VaR Delta Normal a diferentes niveles de confianza con la metodología correspondiente. 
A continuación, se muestran en la Tabla 1 hasta la 4, los resultados anuales de: valor esperado del rendimiento del activo, desviación estándar del activo, beta del activo respecto al mercado, tasa libre de riesgo e índice de Sharpe.

Tabla 1. Resultados de las variables financieras de cada activo para Brasil

\begin{tabular}{|c|c|c|c|c|}
\hline Variable & B3SA3 & BBSE3 & $\ldots$ & BOVESPA \\
\hline$E\left(R_{i}\right)$ & $36.5200 \%$ & $7.7775 \%$ & $\ldots$ & $11.0451 \%$ \\
\hline$\sigma_{i}$ & 0.4648 & 0.3278 & $\ldots$ & 0.3438 \\
\hline$\beta_{i m}$ & 1.0991 & 0.6464 & $\ldots$ & 1.0000 \\
\hline$T L R$ & $2.0000 \%$ & $2.0000 \%$ & $\ldots$ & $2.0000 \%$ \\
\hline$I S$ & 0.7426 & 0.1762 & $\ldots$ & 0.2630 \\
\hline
\end{tabular}

Fuente: Elaboración propia mediante Excel 2016.

La Tabla 1. Muestra que de los 77 componentes que cotizaron a la par del mercado brasileño (BOVESPA) únicamente 51 fueron previamente seleccionados como candidatos para la conformación de portafolios al ser su $I S>0$, algunos de éstos, tuvieron betas mayores a la unidad y otros estuvieron por debajo, se muestra también un valor esperado de dicho mercado positivo mayor a su tasa de referencia SELIC al 12/02/2021.

Tabla 2. Resultados de las variables financieras de cada activo para Argentina

\begin{tabular}{|c|c|c|c|c|c|}
\hline Variable & ALUA & MIRG & TXAR & VALO & $\begin{array}{c}\text { S\&P } \\
\text { Merval }\end{array}$ \\
\hline$E\left(R_{i}\right)$ & $48.8220 \%$ & $65.5263 \%$ & $58.1772 \%$ & $69.5391 \%$ & $15.8693 \%$ \\
\hline$\sigma_{i}$ & 0.5514 & 0.6039 & 0.5649 & 0.5435 & 0.5901 \\
\hline$\beta_{i m}$ & 0.0028 & 0.7068 & 0.6500 & 0.6253 & 1.0000 \\
\hline$T L R$ & $43.8700 \%$ & $43.8700 \%$ & $43.8700 \%$ & $43.8700 \%$ & $43.8700 \%$ \\
\hline$I S$ & 0.0897 & 0.3585 & 0.2532 & 0.4722 & -0.4744 \\
\hline
\end{tabular}

Fuente: Elaboración propia mediante Excel 2016.

La Tabla 2. Muestra que de los 22 componentes que cotizaron a la par del mercado argentino (S\&P Merval) únicamente 4 fueron previamente seleccionados como candidatos para la conformación de portafolios al ser su $I S>0$, todos los activos se muestran con betas menores a la unidad, por lo que son de bajo riesgo, es decir, con poca variabilidad, también se observa un valor esperado de dicho mercado positivo, pero menor a su tasa de política monetaria al 12/02/2021.

Tabla 3. Resultados de las variables financieras de cada activo para Colombia

\begin{tabular}{|c|c|c|c|c|}
\hline Variable & GAA_p & GEB & ISA & COLCAP \\
\hline$E\left(R_{i}\right)$ & $2.7659 \%$ & $16.7609 \%$ & $26.5305 \%$ & $-3.9171 \%$ \\
\hline$\sigma_{i}$ & 0.3442 & 0.2785 & 0.3939 & 0.2673 \\
\hline$\beta_{i m}$ & 0.7604 & 0.4745 & 0.7849 & 1.0000 \\
\hline$T L R$ & $1.8200 \%$ & $1.8200 \%$ & $1.8200 \%$ & $1.8200 \%$ \\
\hline$I S$ & 0.0274 & 0.5363 & 0.6271 & -0.2146 \\
\hline
\end{tabular}

Fuente: Elaboración propia mediante Excel 2016. 
La Tabla 3. Muestra que de los 9 componentes que cotizaron a la par del mercado colombiano (COLCAP) únicamente 3 fueron previamente seleccionados como candidatos para la conformación de portafolios al ser su $I S>0$, todos los activos se muestran con betas menores a la unidad, por lo que son de bajo riesgo, es decir, con poco movimiento, también se observa un valor esperado de dicho mercado negativo, además, su tasa de referencia TES es mayor que éste al 12/02/2021.

Tabla 4. Resultados de las variables financieras de cada activo para México

\begin{tabular}{|c|c|c|c|c|}
\hline Variable & ASURB & BOLSAA & $\ldots$ & S\&P BMV/IPC \\
\hline$E\left(R_{i}\right)$ & $4.2575 \%$ & $11.6176 \%$ & $\ldots$ & $1.7097 \%$ \\
\hline$\sigma_{i}$ & 0.3693 & 0.3743 & $\ldots$ & 0.2019 \\
\hline$\beta_{i m}$ & 1.1019 & 0.7041 & $\ldots$ & 1.0000 \\
\hline$T L R$ & $4.1900 \%$ & $4.1900 \%$ & $\ldots$ & $4.1900 \%$ \\
\hline$I S$ & 0.0018 & 0.1984 & $\ldots$ & -0.1228 \\
\hline
\end{tabular}

Fuente: Elaboración propia mediante Excel 2016.

La Tabla 4. Muestra que de los 30 componentes que cotizaron a la par del mercado mexicano (S\&P BMV/IPC) únicamente 13 fueron previamente seleccionados como candidatos para la conformación de portafolios al ser su $I S>0$, algunos activos se muestran con betas mayores a la unidad, y otros por debajo, también se observa un valor esperado de dicho mercado positivo, pero menor a su tasa de referencia TIIE al 12/02/2021.

El detalle de los portafolios diversificados por cada empresa seleccionada por los modelos de Markowitz y Sharpe para cada país se puede apreciar en el Anexo.

Asimismo, se consideró una inversión inicial de 1 millón de pesos mexicanos para todos los portafolios, Finalmente se homologaron todos los resultados del VaR en dólares considerando un horizonte temporal de un año, como se resume en la Tabla 5 y 6 . Respectivamente.

Tabla 5. Valor en Riesgo en USD a diferentes niveles de confianza para el portafolio de Markowitz

\begin{tabular}{|c|c|c|c|}
\hline & $\begin{array}{c}\text { Markowitz USD } \\
\mathbf{9 5 \%}\end{array}$ & $\begin{array}{c}\text { Markowitz USD } \\
\mathbf{9 9 \%}\end{array}$ & $\begin{array}{c}\text { Markowitz USD } \\
\mathbf{9 9 . 9 \%}\end{array}$ \\
\hline Argentina & $-\$ 29,645.3668$ & $-\$ 41,928.0080$ & $-\$ 55,695.5761$ \\
\hline Colombia & $-\$ 20,082.7876$ & $-\$ 28,403.4698$ & $-\$ 37,730.0924$ \\
\hline Brasil & $-\$ 16,132.8615$ & $-\$ 22,817.0139$ & $-\$ 30,309.2562$ \\
\hline México & $-\$ 14,562.8808$ & $-\$ 20,596.5603$ & $-\$ 27,359.6897$ \\
\hline \multicolumn{4}{|c}{ Fuente: Elaboración propia mediante Excel 2016. }
\end{tabular}

En la Tabla 5. Se observa que las pérdidas esperadas en los portafolios del tipo Markowitz para Argentina son mayores que en todos los demás países de América Latina analizados.

Tabla 6. Valor en Riesgo en USD a diferentes niveles de confianza para el portafolio de Sharpe

\begin{tabular}{|c|c|c|c|}
\hline & $\begin{array}{c}\text { Sharpe USD } \\
\mathbf{9 5 \%}\end{array}$ & $\begin{array}{c}\text { Sharpe USD } \\
\mathbf{9 9 \%}\end{array}$ & $\begin{array}{c}\text { Sharpe USD } \\
\mathbf{9 9 . 9 \%}\end{array}$ \\
\hline Argentina & $-\$ 37,029.1608$ & $-\$ 52,371.0488$ & $-\$ 69,567.7154$ \\
\hline Colombia & $-\$ 29,510.8949$ & $-\$ 41,737.8219$ & $-\$ 55,442.9400$ \\
\hline
\end{tabular}




\begin{tabular}{|c|c|c|c|} 
Brasil & $-\$ 23,203.6419$ & $-\$ 32,817.3536$ & $-\$ 43,593.3282$ \\
\hline México & $-\$ 18,582.0267$ & $-\$ 26,280.9150$ & $-\$ 34,910.5710$ \\
\hline
\end{tabular}

Fuente: Elaboración propia mediante Excel 2016.

En la Tabla 6. Se observa nuevamente que las pérdidas esperadas en los portafolios del tipo Sharpe para Argentina son mayores que en todos los demás países de América Latina.

De las Tablas 5 y 6 también se puede observar con claridad que México es el país que tiene las pérdidas esperadas menores independientemente del tipo de portafolio óptimo y del nivel de significancia establecido.

En las gráficas siguientes se muestran la construcción que engloba todos los portafolios para cada país.

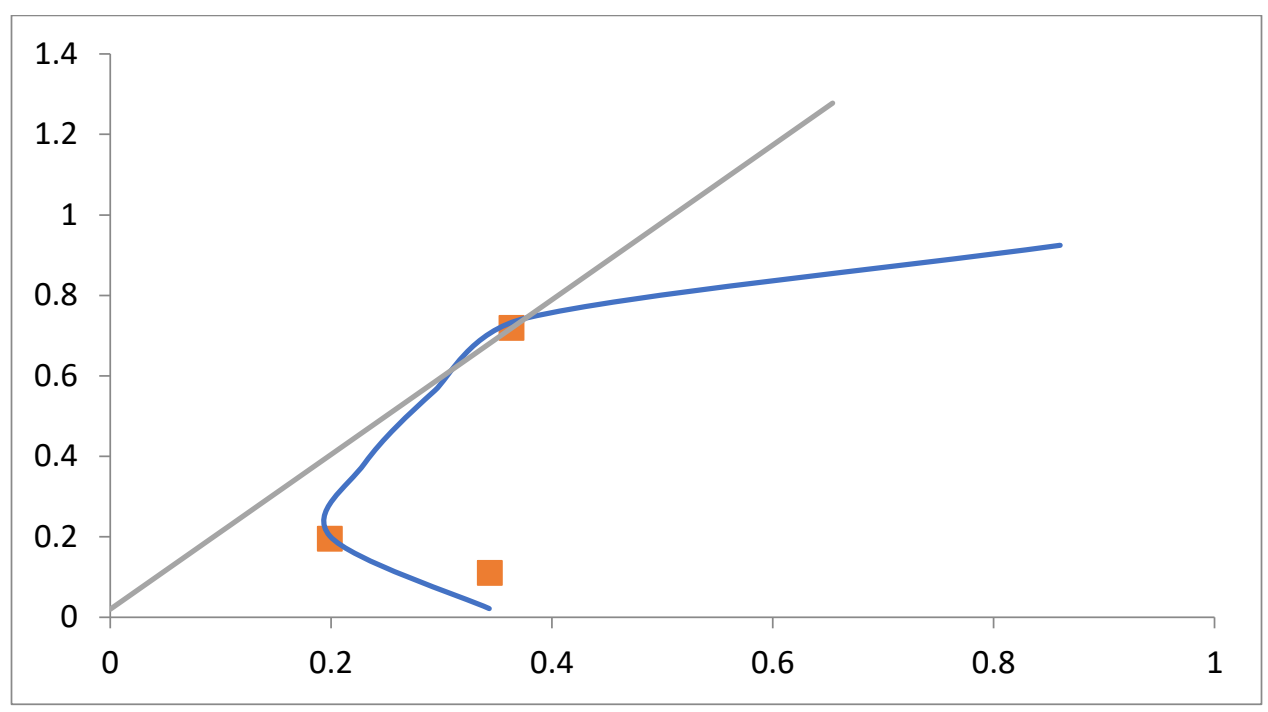

Gráfica 1. Construcción de los portafolios para Brasil.

Fuente: Elaboración propia mediante Excel 2016.

La Gráfica 1. Resume el caso de Brasil, donde se observa que tanto el portafolio de Markowitz cuya beta es de 0.4419 , como el de Sharpe con beta de 0.8438 otorgan mayores rendimientos que su propio índice (BOVESPA), el cuál claramente se puede apreciar con un mayor riesgo respecto al portafolio de Markowitz y un poco menor al de Sharpe pero con rendimientos esperados significativamente menores. 


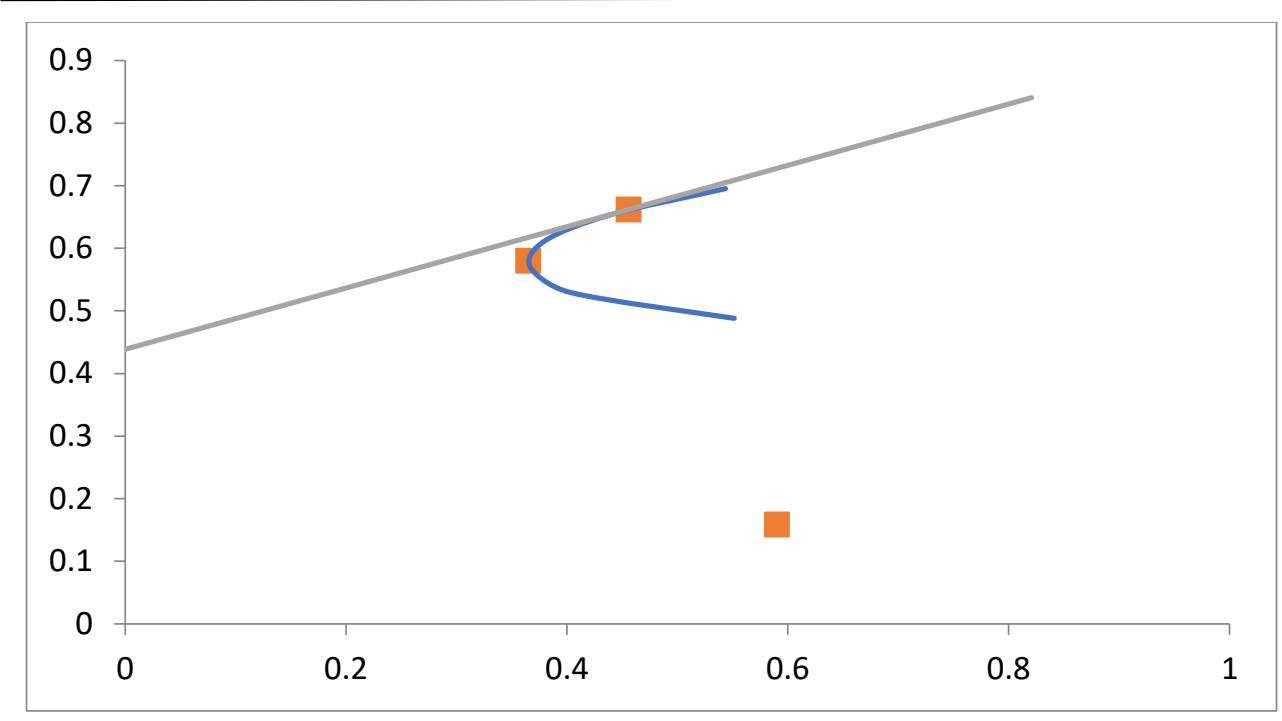

Gráfica 2. Construcción de los portafolios para Argentina

Fuente: Elaboración propia mediante Excel 2016.

La Gráfica 2. Resume el caso de Argentina, donde se observa que tanto el portafolio de Markowitz cuya beta es de 0.3803 , como el de Sharpe con beta de 0.5643 otorgan mayores rendimientos que su propio índice (S\&P MERVAL), el cuál claramente se puede apreciar con un mayor riesgo respecto al portafolio de Markowitz y de Sharpe pero con rendimientos esperados mucho menores.

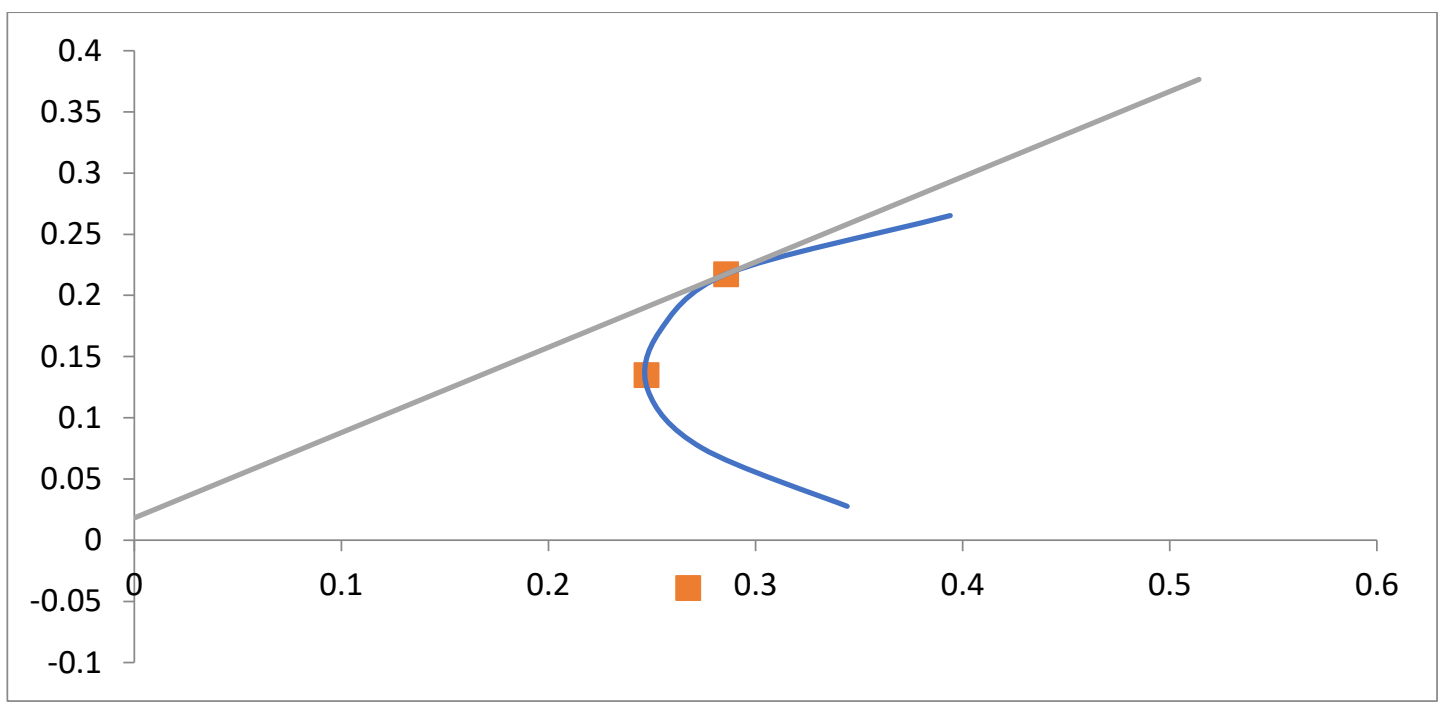

Gráfica 3. Construcción de los portafolios para Colombia.

Fuente: Elaboración propia mediante Excel 2016.

La Gráfica 3. Resume el caso de Colombia, donde se observa que en el portafolio de Markowitz cuya beta es de 0.5889 , prácticamente se tiene el mismo nivel de riesgo que el de su propio índice (COLCAP), pero claramente se observa que es menor respecto al riesgo del portafolio de Sharpe con 
beta de 0.6324 , en ambos casos el rendimiento esperado por el COLCAP es mucho más pequeño que el de ambos portafolios óptimos, de echo es negativo.

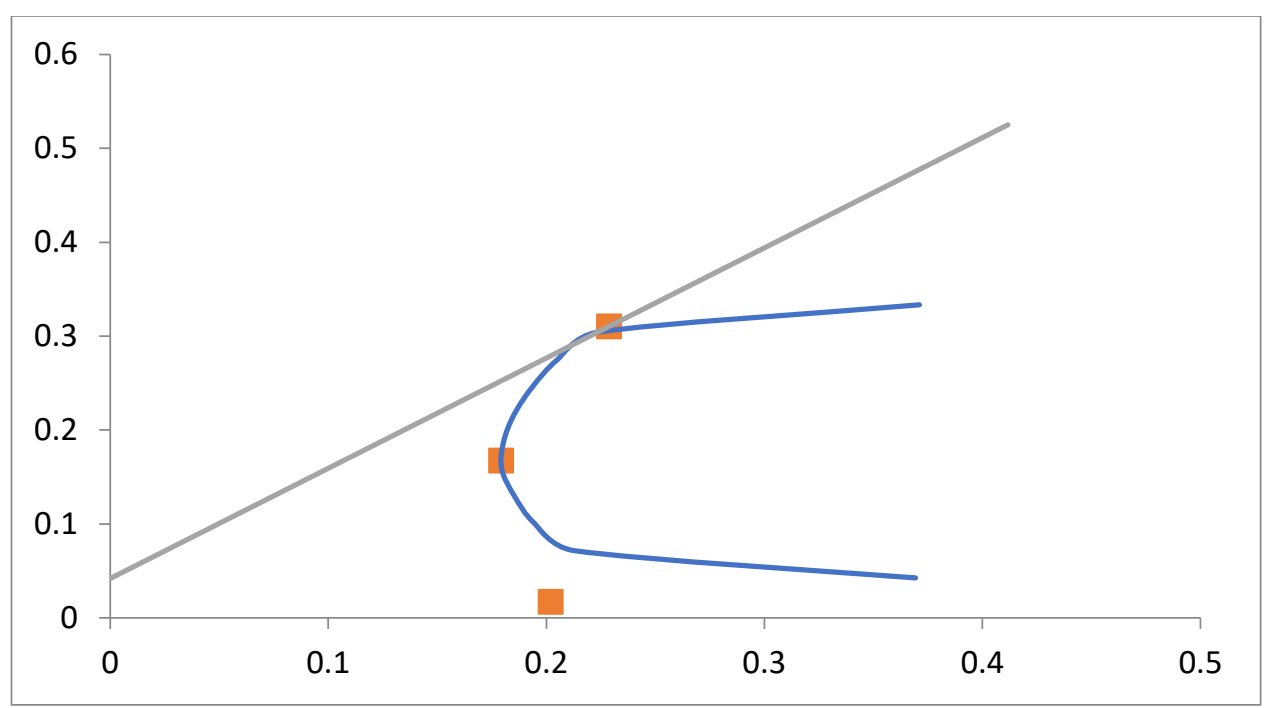

Gráfica 4. Construcción de los portafolios para México.

Fuente: Elaboración propia mediante Excel 2016.

La Gráfica 4. Resume el caso de México, donde se observa que en el portafolio de Markowitz cuya beta es de 0.6455, el riesgo es un poco menor que el de su índice (S\&P BMV/IPC), y ambos son menores respecto al riesgo del portafolio de Sharpe con beta de 0.6815 , en ambos casos el rendimiento esperado por el S\&P BMV/IPC es mucho más pequeño que el de ambos portafolios óptimos.

\section{Conclusiones}

En este estudio, después de realizar un análisis financiero exhaustivo en las economías de América Latina más afectadas por la pandemia de COVID-19 hoy en día, se observa que mediante la métrica de VaR tanto para portafolios óptimos del tipo Markowitz y de Sharpe, los resultados con pérdidas esperadas más alarmantes son para Argentina y los menores para México. Por lo anterior se pudo determinar la afectación que ha causado la pandemia en cada país analizado al menos desde el punto de vista financiero con la métrica mencionada, quizás a simple vista viendo los números que arroja para Brasil la pandemia COVID-19 se pudiera haber pensado intuitivamente que también desde el punto de vista financiero sería el país más afectado. Pero no es así, empíricamente se ha demostrado que lo es Argentina. Por lo cual se cumple tanto el objetivo de la investigación como la hipótesis.

Todos los portafolios analizados mostraron betas menores a la unidad, por lo que son de bajo riesgo. Lo anterior puede ser de utilidad al inversionista que opte por realizar inversiones en alguno de los mercados de América Latina analizados en tiempos de pandemia, sugiriendo tenga un perfil de riesgo conservador, buscando limitar sus pérdidas en vez de tener la expectativa de ganancias extraordinarias. 
Finalmente, como limitación se tiene que los modelos expuestos consideran el supuesto de rendimientos gaussianos, por lo que para futuras investigaciones se puede romper este supuesto para la construcción de portafolios.

\section{Referencias}

[1] Agudelo, G. A., Olivares, H. A., y Téllez, J. (2021). Riesgo de mercado en Portafolios mexicanos previo a la crisis COVID-19: Portafolio de renta fija vs Portafolio de capital. REMEF, vol. 16, núm. 4, pp. 1-21, e520. DOI: https://doi.org/10.21919/remef.v16i4.520

[2] Álvarez, S., Restrepo, D., y Velásquez, M. (2017). Medición del valor en riesgo de portafolios de renta fija usando modelos multifactoriales dinámicos de tasas de interés. Estudios Gerenciales, 33(142), 5263. DOI:https://doi.org/10.1016/j.estger.2017.02.003

[3] Brun, X., y Moreno, M. (2008). Análisis y selección de inversiones en mercados financieros: eficiencia de los mercados, teoría de carteras, asignación de activos y definición de inversión. Barcelona: Bresca: Profit.

[4] Byder, J., Agudelo, D., y Arango, I. (2019). Gender matters most. The impact on short-term risk aversion following and financial crash. Review of Financial Economics, 37(1), 106-117. DOI: https://doi.org/10.1002/rfe.1038

[5] CONAPRENC (2020). Coronavirus, Recuperado de: http://CONAPRENC/posts/coronavirus/2733603850021557/ [Consultado el 30 de abril de 2021]

[6] Estrada, E., y Toro, C. (2014). Valor y riesgo en carteras de inversión, variables críticas calculadas a partir de un sistema híbrido inteligente basado en CBR. Conferencia Ibérica sobre Sistemas y Tecnologías de la Información, CISTI.

[7] Fama, E.F. (1965). Portfolio Analysis in a Stable Paretian Market. Management Science, 11(3), 361490. DOI: https://doi.org/10.1287/mnsc.11.3.404.

[8] García, F., González, J., Oliver, J., y Rueda, G. (2019). Medidas de riesgo en la selección de cartera. Espacios, 40(38), 18-30.

[9] Gutiérrez, M., Gálvez, P., Eltit B., y Reinoso, H. (2017). Resolución del problema de carteras de inversión utilizando la heurística de colonia artificial de abejas. Estudios Gerenciales, 33(145), 391-399. DOI: https://doi.org/10.1016/j.estger.2017.11.001

[10] León, A., Martínez, M., y Garza, L. (2015). Comparación de los enfoques de media-varianza y mediasemivariancia para elegir una cartera agrícola. Revista Chapingo, Serie Horicultura, 21(1), 71-80. DOI: https://doi.org/10.5154/r.rchsh.2014.04.020

[11] Lintner, J. (1965). The Valuation of risky Assets: The Selection of Risky Investments in Stock Portfolios and Capital Budgets. Review of Economics and Statistics, 47(1), 13-37. DOI: https://doi.org/10.2307/1924119

[12] Luna, S., y Agudelo, D. (2019). ¿Agrega valor el modelo Black-Litterman en portafolios del Mercado Integrado Latinoamericano (MILA)?. Revista de Métodos Cuantitativos para la Economía y la Empresa, 27(1), 55-73. DOI: https://doi.org/10.2139/ssrn.3297111

[13] Markowitz, H. (1952). Portfolio Selection. Journal of Finance, 7(1), 77-91. DOI: https://doi.org/10.1111/j.1540-6261.1952.tb01525.x

[14] Martínez, C. y Parraguez, C. (2021). Daño social, neoliberalismo y Pandemia en América latina. Papeles de Población, 27(107), 103-140.

[15] Mendoza-Rivera, R. J., Lozano-Díez, J. A., Venegas-Martínez, F. (2020). Impacto de la pandemia Covid19 en variables financieras relevantes en las principales economías de Latinoamérica. Economía Teoría 
y Práctica, $\quad$ vol. $\quad 28, \quad$ núm. $\quad$ Especial, 125-144. DOI: http://dx.doi.org/10.24275/ETYPUAM/NE/E052020/Mendoza

[16] Mora, M., Franco, J., y Preciado, L. (2010). Asignación óptima de cartera para índices bursátiles latinoamericanos. Cuadernos de Administración, 23(40), 191-214.

[17] [17] Mossin, J. (1966). Equilibrium in a Capital Asset Market. Econometrica: Journal of the econometric society, 34(1), 768-783. DOI: https://doi.org/10.2307/1910098

[18] Olivares, H. A., Bucio, C., Agudelo, G. A., Franco, L. C., y Franco, L. E. (2017). Valor en Riesgo: Un análisis del modelo de cópulas elípticas para el sector de vivienda en México. Espacios, vol. 38, num. 31, 27-52.

[19] Preciado, L., y Camacho, V. (2009). Cómo crear un portafolio de inversión con las opciones que ofrecen los fondos de pensiones voluntarias en colombia: el caso de skandia1. Estudios Gerenciales, 25(113), 229-242. DOI: https://doi.org/10.1016/s0123-5923(09)70097-x

[20] Rossignolo, A. (2019). Basel IV A gloomy future for Expected Shortfall risk models. Evidence from the Mexican Stock Market. REMEF, vol. 14, núm. Especial Aniversario, 559-582. DOI: https://doi.org/10.21919/remef.v14i0.423

[21] Sharpe, W. F. (1963). A Simplified Model for Portfolio Analysis. Management Science, 9(1), 277-293. DOI: https://doi.org/10.1287/mnsc.9.2.277

[22] Sharpe, W. F. (1970). Portfolio Theory and Capital Markets. New York: McGraw Hill. DOI: https://doi.org/10.1080/00137917108902694

[23] Tobin, J. (1958). Liquidity Preference as Behavior Towards Risk. Management Science, 6(1),65-86. DOI: https://doi.org/10.2307/2296205

[24] Treynor, J. I. (1962). Toward a Theory of the Market Value of Risky Assets. Unpublished Manuscript. Finally published in 1989 en Asset Pricing and Portfolio Performance. R. A. Korajkzic. Londres: Risk Books. pp. 15-22.

[25] Useche, A. J. (2015). Construcción de portafolios de inversión desde las finanzas del comportamiento: una revisión crítica. Cuadernos de Administración, 28(51), pp. 11-43. DOI: https://doi.org/10.11144/javeriana.cao28-51.cpif

[26] Worldometers (2021). Coronavirus update, Recuperado de: https://www.worldometers.info/coronavirus/ [Consultado el 30 de abril de 2021]

\section{Anexo}

Tabla A1. Ponderaciones para Brasil mediante el modelo de Markowitz

\begin{tabular}{|c|c|c|c|}
\hline Empresa & Ponderación & $\begin{array}{c}\text { Rendimiento } \\
\text { esperado anual }\end{array}$ & Beta \\
\hline BBSE3 & $9.7831 \%$ & $7.7775 \%$ & 0.6464 \\
\hline RADL3 & $10.2273 \%$ & $40.8946 \%$ & 0.5097 \\
\hline SUZB3 & $10.6672 \%$ & $22.2834 \%$ & 0.4481 \\
\hline TAEE11 & $60.1348 \%$ & $18.9575 \%$ & 0.3882 \\
\hline VIVT3 & $8.9894 \%$ & $10.8533 \%$ & 0.4935 \\
\hline
\end{tabular}

Fuente: Elaboración propia mediante Excel 2016. 
Tabla A2. Ponderaciones para Brasil mediante el modelo de Sharpe

\begin{tabular}{|c|c|c|c|}
\hline Empresa & Ponderación & $\begin{array}{c}\text { Rendimiento } \\
\text { esperado anual }\end{array}$ & Beta \\
\hline BRAP4 & $2.5320 \%$ & $43.5920 \%$ & 0.8649 \\
\hline ENEV3 & $38.1432 \%$ & $69.6290 \%$ & 0.8016 \\
\hline MGLU3 & $12.6811 \%$ & $82.5912 \%$ & 1.1837 \\
\hline RADL3 & $7.3905 \%$ & $40.8946 \%$ & 0.5097 \\
\hline WEGE3 & $39.2533 \%$ & $78.2663 \%$ & 0.8368 \\
\hline
\end{tabular}

Fuente: Elaboración propia mediante Excel 2016.

Tabla A3. Ponderaciones para Argentina mediante el modelo de Markowitz

\begin{tabular}{|c|c|c|c|}
\hline Empresa & Ponderación & $\begin{array}{c}\text { Rendimiento } \\
\text { esperado anual }\end{array}$ & Beta \\
\hline ALUA & $41.5443 \%$ & $48.8220 \%$ & 0.0029 \\
\hline MIRG & $10.1671 \%$ & $65.5263 \%$ & 0.7068 \\
\hline TXAR & $21.7838 \%$ & $58.1772 \%$ & 0.6500 \\
\hline VALO & $26.5048 \%$ & $69.5391 \%$ & 0.6253 \\
\hline
\end{tabular}

Fuente: Elaboración propia mediante Excel 2016.

Tabla A4. Ponderaciones para Argentina mediante el modelo de Sharpe

\begin{tabular}{|c|c|c|c|}
\hline Empresa & Ponderación & $\begin{array}{c}\text { Rendimiento } \\
\text { esperado anual }\end{array}$ & Beta \\
\hline ALUA & $12.3455 \%$ & $48.8220 \%$ & 0.0029 \\
\hline MIRG & $19.4363 \%$ & $65.5263 \%$ & 0.7068 \\
\hline VALO & $68.2182 \%$ & $69.5391 \%$ & 0.6253 \\
\hline
\end{tabular}

Fuente: Elaboración propia mediante Excel 2016.

Tabla A5. Ponderaciones para Colombia mediante el modelo de Markowitz

\begin{tabular}{|c|c|c|c|}
\hline Empresa & Ponderación & $\begin{array}{c}\text { Rendimiento } \\
\text { esperado anual }\end{array}$ & Beta \\
\hline GAA_p & $29.8976 \%$ & $2.7659 \%$ & 0.7605 \\
\hline GEB & $60.7838 \%$ & $16.7609 \%$ & 0.4745 \\
\hline ISA & $9.3186 \%$ & $26.5305 \%$ & 0.7850 \\
\hline \multicolumn{4}{|c}{ Fuente: Elaboración propia mediante Excel 2016.}
\end{tabular}

Tabla A6. Ponderaciones para Colombia mediante el modelo de Sharpe

\begin{tabular}{|c|c|c|c|}
\hline Empresa & Ponderación & $\begin{array}{c}\text { Rendimiento } \\
\text { esperado anual }\end{array}$ & Beta \\
\hline GEB & $49.1443 \%$ & $16.7609 \%$ & 0.4745 \\
\hline ISA & $50.8557 \%$ & $26.5305 \%$ & 0.7850 \\
\hline
\end{tabular}

Fuente: Elaboración propia mediante Excel 2016. 
Tabla A7. Ponderaciones para México mediante el modelo de Markowitz

\begin{tabular}{|c|c|c|c|}
\hline Empresa & Ponderación & $\begin{array}{c}\text { Rendimiento } \\
\text { esperado anual }\end{array}$ & Beta \\
\hline ASURB & $10.6849 \%$ & $4.2575 \%$ & 1.1019 \\
\hline BOLSAA & $8.7979 \%$ & $11.6176 \%$ & 0.7041 \\
\hline CUERVO & $21.6140 \%$ & $31.4888 \%$ & 0.3891 \\
\hline GCC & $24.8722 \%$ & $12.4512 \%$ & 0.6099 \\
\hline KIMBERA & $14.5222 \%$ & $4.4513 \%$ & 0.7065 \\
\hline LABB & $12.4915 \%$ & $28.7033 \%$ & 0.5511 \\
\hline PEOLES & $5.5498 \%$ & $12.8864 \%$ & 0.8031 \\
\hline SITESB1 & $1.4676 \%$ & $28.1173 \%$ & 0.9630 \\
\hline
\end{tabular}

Fuente: Elaboración propia mediante Excel 2016.

Tabla A8. Ponderaciones para México mediante el modelo de Sharpe

\begin{tabular}{|c|c|c|c|}
\hline Empresa & Ponderación & $\begin{array}{c}\text { Rendimiento } \\
\text { esperado anual }\end{array}$ & Beta \\
\hline CUERVO & $44.1196 \%$ & $31.4888 \%$ & 0.3891 \\
\hline GMEXICOB & $25.1399 \%$ & $33.3425 \%$ & 1.1882 \\
\hline LABB & $20.6216 \%$ & $28.7033 \%$ & 0.5511 \\
\hline SITESB1 & $10.1190 \%$ & $28.1173 \%$ & 0.9630 \\
\hline
\end{tabular}

Fuente: Elaboración propia mediante Excel 2016. 